\title{
Manejo e integración estomatológica del paciente pediátrico con trastorno de Asperger.
}

\author{
Management and dental integration of the \\ pediatric patient with Asperger disorder.
}

\author{
Karla Ivette Oliva-Olvera,* Mónica Badillo-Barba,‡ Rodrigo Díaz-Romero,§ \\ Enrique Ensaldo-Carrasco"
}

\section{RESUMEN}

Trastorno neurológico enmarcado dentro de los trastornos de espectro autista (TEA) cuyas manifestaciones se reflejan en los ámbitos de la comunicación, interacción e imaginación social. Se clasifica en el manual diagnóstico y estadístico de trastornos mentales (DSM IV), se caracteriza por un mayor o menor grado de deterioro en las habilidades de lenguaje y comunicación, así como patrones repetitivos o restrictivos de pensamiento y comportamiento. El síntoma más distintivo es el interés obsesivo en un solo objeto o tema y la exclusión de cualquier otro pero siempre conservando habilidades de lenguaje. El pronóstico es bueno, debido a la compensación cognitiva, el enfoque repetitivo y restrictivo a actividades humanas productivas o generadoras de deferencias particulares, aunque no hay tratamiento específico, sino más bien interdisciplinario e individualizado, éste consiste en manejar los síntomas conductuales y la comorbilidad de forma independiente ya sea farmacológica o intervencionista. Paciente masculino de cinco años de edad, con un peso de $26 \mathrm{~kg}$, cuadro de inmunizaciones completas, previamente diagnosticado con trastorno de Asperger (2015); caries dental de diversos grados, manejo estomatológico para su rehabilitación. El objetivo de este reporte es dar a conocer los cuidados para el tratamiento dental en pacientes con este trastorno.

Palabras clave: Trastorno de Asperger, autismo, Rett, manejo conductual, manejo estomatológico.

\section{ABSTRACT}

Neurological disorder known as autism spectrum disorders (ASD) whose main manifestations are reflected in the areas of communication, interaction and social imagination. It was first classified in the Diagnostic and Statistical Manual of Mental Disorders (DSM IV), characterized by a greater or lesser degree of deterioration in language and communication skills, as well as repetitive patterns or restrictive of thought and behavior. The most distinctive symptom is obsessive interest in a single object or topic and the exclusion of any other, but always retaining language skills. The prognosis is good in most of the cases, due to the cognitive compensation, the repetitive and restrictive approach to productive or deferential human activities, although there is no specific treatment, but rather interdisciplinary and individualized, this consists of managing behavioral symptoms and comorbidity independently either pharmacologically or interventionally. Male patient with five years old and weight of $26 \mathrm{~kg}$, complete immunization chart, previously diagnosed with Asperger's disorder (2015); with dental caries of various degrees implementing dental management. The objective of this report is to make aware of the care and behavior management for dental treatment in patients with this Disorder.

Keywords: Asperger disorder, autism, Rett, behavioral management, dental management.

\footnotetext{
* Postgrado en Odontología Pediátrica. Profesor-Investigador. Departamento de Atención a la Salud.

‡ Postgrado en Ortodoncia y Prótesis Dental. Profesor-Investigador. Departamento de Atención a la Salud.

$\S$ Pasante en Laboratorio de diseño y comprobación San Lorenzo Atemoaya; de la Licenciatura en Estomatología.

ๆ Postgrado en Endoperiodontología. Profesor-Investigador. Departamento de Atención a la Salud.
}

Universidad Autónoma Metropolitana, Unidad Xochimilco. México, Ciudad de México.

Recibido: 25 de marzo de 2020. Aceptado: 26 de enero de 2021.

Citar como: Oliva-Olvera KI, Badillo-Barba M, Díaz-Romero R, Ensaldo-Carrasco E. Manejo e integración estomatológica del paciente pediátrico con trastorno de Asperger. Rev ADM. 2021; 78 (2): 100-105. https://dx.doi.org/10.35366/99286 


\section{INTRODUCCIÓN}

$\mathrm{E}^{\prime}$ trastorno de Asperger fue descrito en 1944 por el médico pediatra austriaco Hans Asperger. Este hecho casi coincidió cronológicamente con la publicación en 1943 del doctor Leo Kanner sobre los trastornos autísticos del contacto afectivo, cuyo trabajo dio origen a las ideas modernas del trastorno autista.

Tanto las descripciones de Asperger como las de Kanner se centran en aspectos muy peculiares de la conducta infantil. ${ }^{1}$ Este padecimiento es un trastorno neurológico enmarcado dentro de los trastornos de espectro autista (TEA) cuyas principales manifestaciones se reflejan en los ámbitos de la comunicación, interacción e imaginación social. $^{2}$

En 1994, la Asociación Americana de Psiquiatría (APA) clasificó por primera vez el Trastorno de Asperger (TA) en el manual diagnóstico y estadístico de trastornos mentales (DSM IV) en la categoría de trastornos generalizados del desarrollo, los cuales se definen por las conductas y manifestaciones observables. ${ }^{3}$ Una de las principales características diagnósticas y que definen a los trastornos de espectro autista (TEA) es el deterioro de la interacción social. La falta de competencias sociales asociadas al TEA puede interrumpir el desarrollo, llevando a una disminución en las experiencias de aprendizaje positivo. ${ }^{4}$

Se ha demostrado que las intervenciones basadas en los principios del análisis del comportamiento aplicado mejoran las habilidades de comunicación funcional en niños con TEA y disminuyen comportamientos problemáticos como la agresión, pero las intervenciones terapéuticas dirigidas a déficits sociales no han alcanzado el mismo nivel de atención. De hecho, los déficits sociales en esta población siguen siendo un gran desafío de tratamiento. ${ }^{5}$

La falta de habilidades sociales y la capacidad de determinar cuándo utilizar estas habilidades también representan una discapacidad social general; dado que los niños con TEA no logran adquirir habilidades sociales apropiadas y pueden carecer de oportunidades para interactuar positivamente con quienes frecuentan, una intervención racional sería la capacitación específica llevada a cabo en un formato grupal, es decir, la integración en un grupo podría contribuir en la habilidad del infante para crear relaciones sociales estructuradas y específicas, mejorando así su habilidad para generar lazos interpersonales con individuos ajenos a su núcleo familiar, por ejemplo con el odontólogo, quien pretende brindarle tratamiento.

El trastorno de Asperger afecta el funcionamiento social y el espectro de actividades e intereses. Se ca- racteriza por un mayor o menor grado de deterioro en las habilidades de lenguaje y comunicación, así como patrones repetitivos o restrictivos de pensamiento y comportamiento. El síntoma más distintivo en el trastorno de Asperger es el interés obsesivo en un solo objeto o tema y la exclusión de cualquier otro. A diferencia de otros tipos de autismo, los individuos con trastorno de Asperger conservan sus habilidades de lenguaje temprano. ${ }^{6}$

Las características esenciales del trastorno de Asperger son:

1. La alteración persistente de la interacción social (criterio A).

2. El desarrollo de patrones del comportamiento, intereses y actividades restrictivas y repetitivas (criterio B).

3. El trastorno puede dar lugar a un deterioro clínicamente significativo social, laboral o de otras áreas importantes de la actividad del individuo (criterio C).

4. En contraste con el trastorno autista, no existen retrasos del lenguaje clínicamente significativos (criterio D).

5. Además, no se observan retrasos clínicamente significativos del desarrollo cognoscitivo ni en el desarrollo de habilidades de autoayuda propias de la edad del sujeto, comportamiento adaptativo y curiosidad acerca del ambiente durante la infancia (criterio E).

6. No se establece el diagnóstico si se cumplen criterios de cualquier otro trastorno generalizado del desarrollo específico o de esquizofrenia (criterio F). ${ }^{7}$

Las características anteriores se desglosan en la Tabla 1.

\section{Diagnóstico diferencial}

Resulta evidente que la atención estomatológica dirigida a un paciente que presenta algún trastorno de espectro autista no es propiamente diagnosticada por los profesionales en el ramo. Los pacientes que acuden a consulta regular, y de quienes se sospecha que padecen un trastorno mental, de comportamiento o de integración social, deberán contar con un diagnóstico emitido por el especialista en psiquiatría y psicología tras una serie de estudios conductuales y neuropsiquiátricos que identifiquen el trastorno que presenta un paciente, así como su grado de severidad, evolución y pronóstico.

Resulta de gran importancia conocer los diagnósticos diferenciales relacionados con los trastornos de espectro autista, pues esto ayudará a orientar el plan de tratamiento y cubrir las necesidades de cada paciente, ya sea que cuente o no con un diagnóstico neuropsiquiátrico. 
El trastorno de Asperger no se diagnostica si se cumplen criterios de otro trastorno generalizado del desarrollo o de esquizofrenia.

El trastorno autista puede distinguirse del trastorno de Asperger por la ausencia de retraso en el desarrollo del lenguaje; por lo tanto no se diagnostica como tal si cumple con criterios del trastorno autista.

El trastorno de Rett difiere del trastorno desintegrativo infantil y del trastorno de Asperger en su proporción sexual característica, su inicio y su patrón de déficit. El trastorno de Rett sólo ha sido diagnosticado en mujeres, mientras que el trastorno desintegrativo infantil y el trastorno de Asperger parecen ser más frecuentes en varones. El trastorno de Rett es un desorden neurogenético caracterizado por un periodo típico de desarrollo seguido por una regresión significativa en las habilidades, incluyendo habilidades motrices, comunicación y movimientos útiles de las manos. ${ }^{8}$

El trastorno de Asperger y el trastorno obsesivo-compulsivo comparten unos patrones del comportamiento repetitivo y estereotipado. A diferencia del trastorno obsesivo-compulsivo, el trastorno de Asperger se caracteriza por una alteración cualitativa de la interacción social y un patrón de intereses y actividades más restrictivo.

\section{Curso del trastorno}

El retraso motor o la torpeza motora pueden observarse durante el periodo preescolar. Las deficiencias en la interacción social pueden ponerse de manifiesto en la vida escolar, durante esta época es cuando pueden aparecer o reconocerse los peculiares intereses circunscritos o idiosincrásicos.

En la vida adulta, los sujetos con este trastorno pueden experimentar problemas relacionados con la empatía y la modulación de la interacción social. Aparentemente, este trastorno sigue un curso continuo y, en la mayor parte de los casos, se prolonga durante toda la vida.

\section{Manejo estomatológico}

Estos pacientes requieren cuidados determinados, así como técnicas conductuales específicas para poder realizar el tratamiento dental. Al presentar un cuadro complejo, es necesario hacer un abordaje integral e interdisciplinario donde sean tomadas en cuenta sus características biológicas, psicológicas y sociales, aunadas a un examen del sistema estomatognático a fin de brindar una adecuada atención en la consulta odontológica.

Tabla 1: Criterios diagnósticos considerados en el trastorno de Asperger; DSM IV.

Criterios para el diagnóstico de F84.5 trastorno de Asperger [299.80]

1. Alteración cualitativa de la interacción social, manifestada al menos por dos de las siguientes características:

a. Importante alteración del uso de múltiples comportamientos no verbales como contacto ocular, expresión facial, posturas corporales y gestos reguladores de la interacción social

b. Incapacidad para desarrollar relaciones con compañeros apropiadas al nivel de desarrollo del sujeto

c. Ausencia de la tendencia espontánea a compartir disfrutes, intereses y objetivos con otras personas (p. ej: no mostrar, traer o enseñar a otras personas objetos de interés)

d. Ausencia de reciprocidad social o emocional

2. Patrones de comportamiento, intereses y actividades restrictivos, repetitivos y estereotipados, manifestados al menos por una de las siguientes características:

a. Preocupación absorbente por uno o más patrones de interés estereotipados y restrictivos que son anormales, sea por su intensidad, sea por su objetivo

b. Adhesión aparentemente inflexible a rutinas o rituales específicos, no funcionales

c. Manierismos motores estereotipados y repetitivos (p. ej: sacudir o girar manos o dedos o movimientos completos de todo el cuerpo)

d. Preocupación persistente por partes de objetos

3. El trastorno causa un deterioro clínicamente significativo de la actividad social, laboral y otras áreas importantes de la actividad del individuo

4. No hay retraso general del lenguaje clínicamente significativo (p. ej: a los dos años de edad utiliza palabras sencillas, a los tres años de edad utiliza frases comunicativas)

5. No hay retraso clínicamente significativo del desarrollo cognoscitivo ni del desarrollo de habilidades de autoayuda propias de la edad, comportamiento adaptativo (distinto de la interacción social) y curiosidad acerca del ambiente durante la infancia

6. No cumple los criterios de otro trastorno generalizado del desarrollo ni de esquizofrenia 
La forma ideal de asistencia odontológica a casos con necesidades especiales está estratificada en tres niveles: ${ }^{9}$

Nivel 1

1. Asistencia en atención primaria.

2. Captación del paciente con discapacidad.

3. Educación en salud bucodental a los responsables del paciente.

4. Evaluación el estado bucodental.

5. Evaluación de la conducta.

6. Establecimiento de un plan de tratamiento bucodental provisional.

7. Tratamientos odontológicos sencillos.

Nivel 2

1. Asistencia especializada ambulatoria.

2. Reevaluación del paciente.

3. Tratamientos dentales complejos que no requieran anestesia general.

Nivel 3

1. Asistencia odontológica hospitalaria.

2. Reevaluación del paciente.

3. Tratamientos dentales complejos que requieran anestesia general.

4. Tratamientos dentales en pacientes de alto riesgo médico.

El éxito de un tratamiento en un paciente con necesidades especiales es el acondicionamiento a la consulta, ya que mediante un adecuado abordaje conductual, el manejo de este se facilitará notablemente.

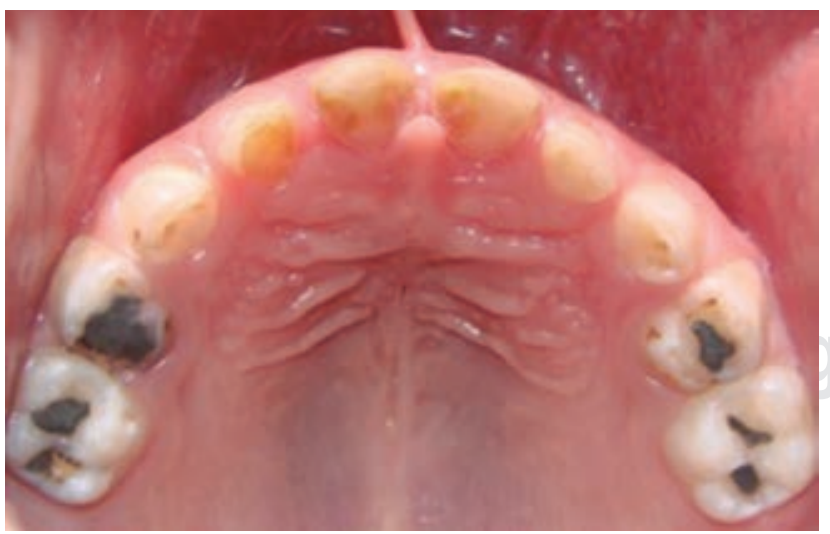

Figura 1: Paciente con síndrome de Asperger: fotografía inicial; arcada superior.

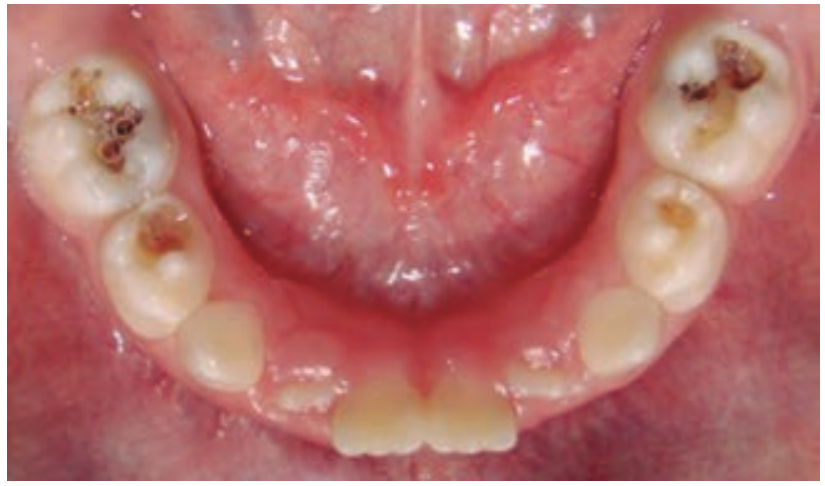

Figura 2: Paciente con síndrome de Asperger: fotografía inicial; arcada inferior.

Para prevenir y evitar la aparición de patología bucodental, así como para su rehabilitación, se hace necesario un plan de salud bucodental completo e integrado en todos sus niveles, siendo indispensable la acción de la atención primaria odontológica, así como la atención especializada.

Es muy importante alentar la prevención dental, con el objetivo de cambiar problemas de conducta o utilizar técnicas para familiarizar al sujeto con la higiene bucal y proveer entrenamientos y técnicas a los padres.

En casos con discapacidades del desarrollo, lo básico es trabajar la correcta técnica de cepillado, prevenir enfermedades bucales futuras, ya que estas personas pueden no ser autosuficientes y algunas veces dependen de proveedores de cuidado para cepillar sus dientes todos los días.

Suelen presentar mucha dificultad con las transiciones; tener una representación gráfica o un cronograma de actividades suele ser provechoso. Se debe proporcionar tanta información previa a la transición como sea posible si va a haber un cambio o una modificación en el horario, y proporcionar una o dos advertencias previas a un cambio de actividad. El uso de claves e indicaciones visuales resulta de gran ayuda, también hablar lentamente y con frases más cortas.

Las instrucciones se entienden más fácilmente si se repiten con claridad y de forma sencilla. Este tipo de paciente puede actuar de una manera muy torpe; también puede reaccionar sobreestimulado o muy fuertemente a ciertos gustos, sabores y ruidos fuertes, texturas, olores y luces, debido a una hipersensibilidad a estas situaciones.

Las señales con las manos pueden ser provechosas, especialmente para reforzar ciertos mensajes tales como «deja de hablar», «espera tu turno» o «habla más lenta o suavemente». 
Ocasionalmente, parecerá como si el paciente no está escuchando a pesar de que sí lo está haciendo. No se debe asumir esto, porque el hecho de que no está mirando fijamente al dentista no significa que no lo está oyendo. A diferencia de la mayoría de las personas, a veces forzar el contacto visual rompe su concentración. Él puede oír y entender realmente mejor, si no es forzado a mirar directamente a sus ojos.

\section{PRESENTACIÓN DEL CASO}

Paciente masculino de cinco años siete meses de edad, peso de $26 \mathrm{~kg}$, cuadro de inmunizaciones completas, previamente diagnosticado con síndrome de Asperger en el 2015; presenta caries dental de diversos grados.

En la arcada maxilar presenta múltiples pigmentaciones consistentes con el diagnóstico de caries de grados I, II, III y IV. En los molares temporales, presenta restauraciones desajustadas con obturación de amalgama que presentan filtración de caries (Figura 1).

En la arcada inferior presenta evidentes lesiones cariosas en los órganos dentales 74, 75, 84 y 85, en estos últimos se denotan lesiones más avanzadas (Figura 2).

Se rehabilita bucalmente en varias citas utilizando técnicas de modificación de conducta y comunicación, orientadas en la teoría cognoscitiva de Piaget; la técnica de modificación conductual se basa en la motivación por medio del refuerzo positivo y razonamiento en la etapa preoperacional, la cual consiste en la imitación de ob-

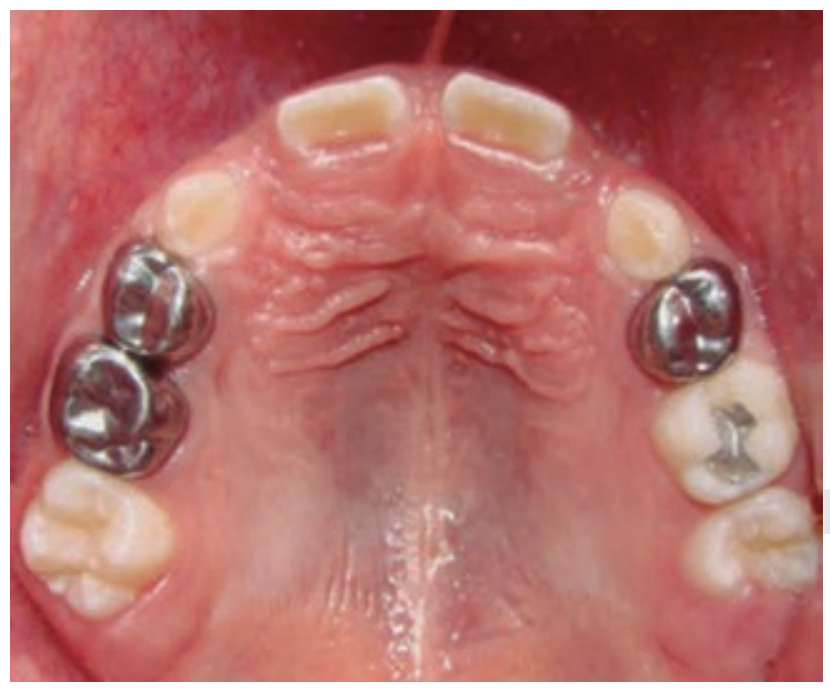

Figura 3: Paciente con síndrome de Asperger: fotografía final; arcada superior.

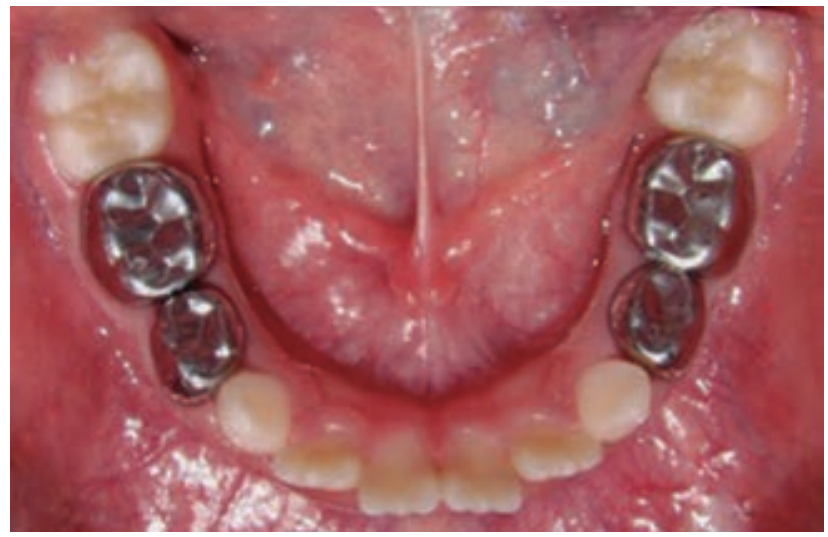

Figura 4: Paciente con síndrome de Asperger: fotografía final; arcada inferior.

jetos de conducta, juegos simbólicos, dibujos, imágenes mentales y el desarrollo del lenguaje hablado, llevándose a cabo tanto en casa como en el consultorio, durante las consultas dentales nos percatamos que el paciente tenía cierto interés en objetos de destreza, por lo que se le prestó un cubo Rubik y se implementó el uso de audífonos con música relajante, dichas tácticas funcionaron de manera positiva en citas cortas, dando como resultado un plan de tratamiento largo. Como complemento de la integración al tratamiento dental, también se dieron consejos para mejorar la dieta e higiene bucal, sin complicaciones durante el tratamiento estomatológico, el cual se terminó meses después notando evidente cambio de dentición (Figuras 3 y 4).

\section{DISCUSIÓN}

La capacidad de los profesionales de la salud para proporcionar el servicio necesario se puede mejorar notablemente con una comprensión de las discapacidades que enfrentan sus pacientes, por tanto, es indispensable la empatía y motivación hacia el paciente con síndrome de Asperger, crear confianza entre profesional-pacientetutor, modificar el entorno con base en el conocimiento, no sólo del trastorno, sino del caso en particular, para brindar la atención e integración de los procedimientos estomatológicos implementados.

\section{CONCLUSIONES}

De acuerdo con la revisión bibliográfica y experiencia en este caso en particular, es de vital importancia dominar cinco puntos importantes: 
1. Integración: modificación de conducta y comunicación con el fin de llevar a cabo los tratamientos necesarios para la rehabilitación integral del paciente con trastorno de Asperger.

2. Motivación: refuerzo positivo y razonamiento en etapa preoperacional.

3. Concientización: consiste en señalar la importancia de la atención estomatológica dirigida a la familia e individuo, ya que sin esta etapa, el tratamiento no mostraría un avance concreto.

4. Higiene: refuerzo de técnicas de cepillado y auxiliares para la higiene bucal.

5. Control: por medio de citas subsecuentes.

\section{REFERENCIAS}

1. Zúñiga MM. El síndrome de Asperger y su clasificación. Educación. 2009; 33 (1): 183-186.

2. Santiso DV. Abordaje integral del síndrome de Asperger. Una perspectiva actual. FMC. 2015; 22 (4): 182-187.

3. Smith O, Jones SC. 'Coming Out' with Autism: Identity in People with an Asperger's Diagnosis After DSM-5. J Autism Dev Disord. 2020; 50 (2): 592-602.

4. O'Connor E. The use of 'Circle of Friends' strategy to improve social interactions and social acceptance: a case study of a child with
Asperger's syndrome and other associated needs. NASEN. 2016; 31: 138-147.

5. White S, Keoning K, Schaill L. Social skills development in children with autism spectrum disorders: a review of the intervention research. J Autism Dev Disord. 2006; 37 (10): 1858-1868.

6. Waldman HB, Perlman SP. Dental assistants and the care of individuals with disabilities: part I. Dent Assist. 2013; 82 (1): 34-35.

7. American Psychiatric Association. Diagnostic and statistical manual of mental disorders, 4th ed., Washington, DC: American Psychiatric Association. 2000.

8. Reichow B, Puskar A, Lutz T, Smith I, Volkmar F. Brief report: systematic review of Rett syndrome in males. J Autism Dev Disord. 2015; 45 (10): 3377-3383.

9. Silvestre DFJ, Plaza CA. Odontología en pacientes especiales. Valencia: Publicaciones de la Universidad de Valencia, España, 2007.

\section{Correspondencia: \\ C.D.E. Karla Ivette Oliva Olvera \\ E-mail:dra_ka_oliva@hotmail.com}

Conflicto de intereses: Los autores declaran no tener ningún conflicto de intereses.

Aspectos éticos: Ninguno.

Financiamiento: Ninguno. 